\title{
tic\&société
}

Vol. 12, $\mathrm{N}^{\circ} 1$ | 1er semestre 2018

Communs numériques et communs de la connaissance

\section{La gouvernance de Wikipédia : élaboration de règles et théorie d'Ostrom}

\section{Gilles SAHUT}

\section{OpenEdition}

\section{Journals}

Édition électronique

URL : https://journals.openedition.org/ticetsociete/2426

DOI : $10.4000 /$ ticetsociete.2426

\section{Éditeur}

Association ARTIC

\section{Édition imprimée}

Pagination : 167-200

\section{Référence électronique}

Gilles SAHUT, «La gouvernance de Wikipédia : élaboration de règles et théorie d'Ostrom », tic\&société [En ligne], Vol. 12, N 1 | 1er semestre 2018, mis en ligne le 31 mai 2018, consulté le 21 septembre 2021. URL : http://journals.openedition.org/ticetsociete/2426 ; DOI : https://doi.org/10.4000/ ticetsociete. 2426 
tic\&société - 12(1), 2018

\title{
La gouvernance de Wikipédia : élaboration de règles et théorie d'Ostrom
}

\begin{abstract}
Gilles SAHUT
Certifié en documentation, Gilles Sahut a été, durant une quinzaine d'années, responsable de la formation des professeurs documentalistes au sein de l'IUFM Midi-Pyrénées, puis de l'École supérieure du professorat et de l'éducation de Toulouse, université de Toulouse 2. II est également docteur qualifié en sciences de l'information et de la communication et chercheur au Lerass (Laboratoire d'études et de recherches appliquées en sciences sociales, Université de Toulouse 3). Ses recherches portent sur la crédibilité de Wikipédia, le rapport des jeunes à cette source et les processus d'élaboration des règles rédactionnelles au sein de cette communauté épistémique. gsahut@univ-tlse2.fr
\end{abstract}




\section{La gouvernance de Wikipédia : élaboration de règles et théorie d'Ostrom}

Résumé : La réussite de Wikipédia est fréquemment attribuée à la pertinence de sa gouvernance. Toutefois, il n'existe pas de consensus scientifique pour la caractériser. Dans cette étude empirique, nous nous penchons sur une facette de cette gouvernance au sein de la Wikipédia francophone : les modalités de construction de deux règles liées à la citation des sources. Elles sont étudiées au travers de la théorie d'Ostrom sur les communs. Nous montrons que ces règles sont discutées et écrites par une minorité de contributeurs particulièrement impliqués. Ainsi, il n'y a pas, dans Wikipédia, de "classe politique " coupée du terrain. Nous soulignons également l'influence du dispositif communicationnel interne sur ce processus ainsi que celle de la Wikipédia anglophone.

Mots-clés: Wikipédia, gouvernance, règles, biens communs, théorie d'Ostrom.

Abstract: The success of Wikipedia is frequently attributed to the relevance of its governance. However, there is no scientific consensus to characterize it. In this empirical study, we examine a facet of this governance: the modalities of construction of two rules related to the citation of sources. We show that these rules are discussed and written by a minority of contributors who are particularly involved. Thus, in Wikipedia, there is no "political class" cut off from the ground. The modalities for the elaboration of the two rules are studied and discussed using Ostrom's theory of the Commons.

Keywords: Wikipedia, governance, policies, commons, Ostrom's theory of the Commons.

Resumen: A menudo, se atribuye el éxito de Wikipedia a la pertinencia de su gobernanza. Sin embargo, no existe un consenso científico para caracterizarla. En este estudio empírico, 
La gouvernance de Wikipédia : élaboration de règles et théorie d'Ostrom

se examina una faceta de esta gobernanza: los tipos de elaboración de dos normas relativas a la citación de las fuentes. Se muestra cómo estas reglas son debatidas y escritas por una minoría de contribuyentes especialmente implicados. Así, en Wikipedia no hay "clase política" alejada de la realidad. Los tipos de elaboración de esas dos normas son estudiados utilizando la teoría de Ostrom sobre los Bienes Comunes.

Palabras clave: Wikipedia, gobernanza, normas, Bienes Comunes, teoría de Ostrom. 


\section{Introduction}

"Le miracle Wikipédia ». Le titre du livre récent de Kaplan et Nova (2016) reflète l'étonnement suscité par la réussite de l'encyclopédie créée par Jimmy Wales en 2001. On peut en effet remarquer que des projets encyclopédiques collaboratifs similaires situés avant cette date (Interpedia, Distributed Encyclopedia, GNUpedia) ou après celle-ci (Citizendium, Knol...) n'ont pas connu le même succès. Pour beaucoup d'analystes (Benkler, 2009; Cardon et Levrel, 2009 ; Fallery et Rodhain, 2013 ; Safner, 2016 ; Viégas, Wattenberg et McKeon, 2007), le facteur clé de la réussite de Wikipédia réside dans son mode de gouvernance, lequel emprunte un certain nombre de traits à la gestion des biens communs naturels étudiés par Elinor Ostrom (2010). Considérée comme un commun de la connaissance, Wikipédia est à la fois regardée comme une expérience politique originale représentative de la capacité d'auto-organisation des collectifs et une promesse démocratique qui pourrait inspirer la gouvernance d'autres domaines de la société (Beaude, 2015).

Pour autant, il n'existe pas de qualification unanime de cette gouvernance. Des essayistes l'ont tour à tour cataloguée comme libertaire, libérale, fasciste, communiste et maoïste. Souvent utilisées pour dénigrer Wikipédia, ces étiquettes ont révélé une méconnaissance de ses mécanismes spécifiques (Jemielniak, 2014). La gouvernance du projet a également fait l'objet de recherches en sciences de l'information, de la communication et en sociologie. Des chercheurs ont mis en avant sa dimension démocratique (Langlais, 2014), adhocratique (Konieczny, 2010), méritocratique (Cardon, 2015), bureaucratique (Butler, Joyce et Pike, 2008) ou encore oligarchique (Canivenc, 2009) ${ }^{1}$, ces

\footnotetext{
${ }^{1}$ Ainsi que le précisent les différents auteurs, ces caractérisations de la gouvernance de Wikipédia doivent être prises comme des idéaux-types et non comme des descriptions absolument fidèles. Par exemple, si Cardon ou Langlais évoquent respectivement l'hypothèse d'une dimension méritocratique ou démocratique de Wikipédia, ils relativisent également la pertinence de ces classifications (par exemple en insistant sur le caractère dynamique de cette école des « ignorants » ou sur le " bricolage politique » wikipédien).
} 
La gouvernance de Wikipédia :

élaboration de règles et théorie d'Ostrom

qualifications n'apparaissant pas toutes complètement compatibles. II semble donc que des études empiriques ciblées sur les différents aspects de la gouvernance wikipédienne pourraient aider à mieux la comprendre et à cerner plus précisément ses particularités. De même, nous avons relevé que ce type d'études demeurait relativement rare pour la Wikipédia francophone, la littérature scientifique internationale étant essentiellement centrée sur la version anglaise de l'encyclopédie.

En ce sens, nous présentons une étude empirique de la genèse et de l'évolution de deux règles de l'encyclopédie en langue française en nous focalisant tout d'abord sur le rôle et le profil des différents acteurs y ayant participé. Ensuite, nous discutons nos résultats et proposons une analyse plus globale des modes de communication et de prise de décision à l'œuvre en nous référant à la théorie des biens communs d'Ostrom. Avant cela, nous dressons un état de la question sur la gouvernance de Wikipédia et exposons le cadre méthodologique de notre étude empirique.

\section{Revue de littérature sur la gouvernance de Wikipédia}

Wikipédia peut être considérée comme un commun de la connaissance au sens où son élaboration et sa gestion échappent à la fois au pouvoir étatique et aux logiques de marché. La ressource collectivement produite est protégée des enclosures par la licence Creative Commons, dont la viralité garantit la pérennité de l'usage libre et ouvert (Le Crosnier, 2015). L'idéal d'accès et de partage des connaissances à l'échelle mondiale inscrit de fait Wikipédia dans la problématique des communs de même que la capacité de la communauté wikipédienne à s'autogouverner.

\subsection{Le parallèle avec la gouvernance des biens communs}

Le terme de gouvernance est ici employé pour désigner l'organisation des mécanismes de contrôle et d'orientation des pratiques rédactionnelles et relationnelles au sein de l'encyclopédie. II englobe la définition des structures politiques et organisationnelles, des rôles formels et des règles officielles. Dans 
une acception plus large, il intègre aussi leurs mises en œuvre de même que les mécanismes informels de régulation qui régissent les comportements wikipédiens (Schroeder et Wagner, 2012). Dès les années 2000 , la recherche a mis en évidence la similitude des modes de gouvernance de Wikipédia avec les principes d'Ostrom régissant la gestion efficace et juste des biens communs naturels (prairies, forêts, système d'irrigation, zones de pêche...). Sept des huit principes énoncés par la politiste américaine peuvent être transposés à l'encyclopédie collaborative :

- La congruence des règles avec la nature de la ressource commune ( $2^{\mathrm{e}}$ principe). Pour illustrer ce principe, Viégas, Wattenberg, Kriss et Van Ham (2007) ont relevé la capacité des wikipédiens à créer des outils adaptés aux problèmes rencontrés. En ce sens, ils ont mentionné la mise au point de l'extension Cite qui, intégrée au dispositif d'édition Mediawiki, facilite l'intégration de notes bibliographiques de bas de page.

- La participation de tous à l'élaboration des règles ( $3^{\mathrm{e}}$ principe). D'après Forte, Larco, et Bruckman (2009), les règles wikipédiennes ont été progressivement précisées par la communauté.

- La surveillance mutuelle des participants (4 ${ }^{\mathrm{e}}$ principe) est facilitée par la transparence des opérations éditoriales (Benkler, 2009 ; Viégas, Wattenberg et McKeon, 2007). Des outils techniques tels que la liste de suivi, qui permet à chaque contributeur de suivre les évolutions d'un article ou encore le LiveRC, qui favorise le contrôle des dernières modifications de l'encyclopédie par des "patrouilleurs" wikipédiens, spécialisés dans cette tâche. Cette distribution de la surveillance participe grandement à la protection de la ressource contre les dégradations volontaires - appelées " vandalisme " - et à la confiance mutuelle entre les participants (Cardon et Levrel, 2009). Les bots, agents automatiques intégrés dans la plateforme d'édition, constituent une spécificité de Wikipédia par rapport aux communs traditionnels étudiés par Ostrom. Ces logiciels effectuent des tâches répétitives à la place 
La gouvernance de Wikipédia :

élaboration de règles et théorie d'Ostrom

des contributeurs, comme l'annulation de certains types de vandalismes. L'activité de surveillance est donc partagée entre les contributeurs et des artefacts, ceux-ci étant considérés comme des acteurs sociaux impliqués dans le projet (Joud, Jullien et Le Gall-Ely, 2016).

- Le système de sanctions graduelles ( $5^{\mathrm{e}}$ principe). II existe dans Wikipédia une grammaire des fautes associée à un design de la sanction qui sépare clairement celles qui sont dirigées contre les contenus de celles qui sont appliquées contre les personnes (Cardon et Levrel, 2009; Safner, 2016).

- Des mécanismes de résolution des conflits facilement accessibles ( $6^{\mathrm{e}}$ principe). Des procédures " en étages », intégrant discussion et médiation, canalisent les disputes et favorisent le maintien d'une cohésion communautaire (Auray, Hurault-Plantet, Poudat et Jacquemin, 2009; Broudoux, 2015)

- Une reconnaissance minimale des droits d'organisation de la communauté ( $7^{\mathrm{e}}$ principe). II faut là clairement distinguer Wikimédia France de la communauté wikipédienne. La première est une association loi 1901 qui a pour mission de promouvoir le « libre partage de la connaissance ${ }^{2}$ " et de soutenir le développement de Wikipédia et des projets connexes (Wikimedia Commons...). La communauté ellemême n'a pas de statut juridique, ce qui ne l'empêche pas de s'autogouverner en s'appuyant sur les principes fondamentaux du projet appelés les "Cinq piliers " ainsi que de très nombreuses règles internes.

- Le $8^{\mathrm{e}}$ principe d'Ostrom précise que, dans les systèmes collectifs de grande taille, il existe une imbrication de différents niveaux organisationnels. Dans Wikipédia, la création de projets spécialisés dans une thématique particulière témoigne d'un certain niveau de décentralisation inhérent à la croissance de l'encyclopédie (Forte, Larco et Bruckman, 2009).

\footnotetext{
${ }^{2}$ Voir l'association Wikimédia France : https://www.wikimedia.fr/lassociation/.
} 
L'application de ces différents principes est donnée comme un facteur de réussite du projet wikipédien qui a conservé son unité tout en ayant connu une croissance forte à la fois du nombre de ses participants et de la ressource construite en commun. Toutefois, cette convergence entre gouvernance de l'encyclopédie et celle des ressources naturelles ne semble pas complète. Le premier principe d'Ostrom indique que des frontières claires entre les membres de la communauté ayant le droit d'utiliser la ressource et ceux qui n'en font pas partie doivent être établies. Le problème se pose différemment pour le document collaboratif qu'est Wikipédia. D'une part, son usage n'est pas réservé à ses contributeurs, ceux-ci ne formant qu'une minorité de ses lecteurs. D'autre part, les degrés de participation à l'activité éditoriale sont extrêmement variables. D'après une étude publiée en 2006 portant sur l'encyclopédie francophone, seuls $5 \%$ des participants ont rédigé $90 \%$ du corpus encyclopédique (Barbe, 2006). Notre propre calcul statistique montre que, de la création du projet à 2015 , moins de $1 \%$ des wikipédiens ont effectué plus de $80 \%$ des modifications de l'encyclopédie, ce qui tend à montrer l'existence d'un noyau communautaire central (Sahut, 2015). Cependant, l'activité des contributeurs occasionnels n'est pour autant pas négligeable et le flou persiste quant à leur appartenance communautaire.

L'application du principe $n^{\circ} 3$ d'Ostrom (la participation de tous à l'élaboration des règles) au projet wikipédien nécessite également d'être discutée. En effet, une pluralité d'acteurs est susceptible de jouer un rôle dans ce processus.

\subsection{Les divers acteurs de la gouvernance}

Le leadership de Wales est fréquemment appréhendé au travers de la figure, non dénuée d'ironie, du «dictateur bienveillant » que l'on retrouve également dans d'autres communautés du logiciel libre comme Linux (Jemielniak, 2014). Durant les premières années du projet, Wales est intervenu directement dans la Wikipédia anglophone pour décider des orientations réglementaires. Sa légitimité provient de son rôle de créateur de l'encyclopédie et de sa société Bomis, qui a fourni un 
La gouvernance de Wikipédia :

élaboration de règles et théorie d'Ostrom

important soutien logistique pour son développement.

En 2003, Wales a fondé la Wikimedia Foundation, une organisation américaine à but non lucratif à laquelle il transfère la propriété du nom de domaine et les serveurs qui hébergent l'encyclopédie. Outre ses missions promotionnelles, financières et techniques, celle-ci édicte des règles qui concernent la dimension juridique du projet. Wales est le président de cette association de 2003 à 2006 et conserve par la suite un siège réservé dans le Board of Trustees, instance décisionnelle de l'organisation.

Différents rôles formels ont émergé au fil de l'histoire de l'encyclopédie. Nous nous contenterons d'évoquer les administrateurs dont le rôle est jugé crucial dans le fonctionnement du projet (Butler, Joyce et Pike, 2008). Élus à l'occasion d'un vote communautaire et choisis parmi les contributeurs expérimentés, ils disposent d'une autorité administrative ${ }^{3}$. D'un point de vue statutaire, leur pouvoir réside uniquement dans l'application des règles en vigueur et des consensus communautaires et non dans leur élaboration. En janvier 2014, ils sont 171 à avoir ce statut dans la Wikipédia francophone, soit 1,1\% des contributeurs actifs (Sahut, 2015).

\subsection{Les principes d'élaboration des règles wikipédiennes}

Dans la présente étude, nous nous focalisons sur l'élaboration des règles qui constitue un aspect central de la gouvernance de Wikipédia. Comme cela est rappelé à plusieurs reprises dans les pages réglementaires de l'encyclopédie, le consensus est le processus décisionnel politique privilégié par les wikipédiens. II n'est pas synonyme d'unanimité communautaire, mais plutôt d'une décision réglementaire acceptée par une large majorité. Wales, qui a promu ce principe dès 2001, s'est inspiré de la notion de rough consensus (consensus approximatif) qui s'est imposée au sein de I'IETF (Internet Engineering Task Force) (Russell, 2006). "We

\footnotetext{
${ }^{3}$ II est à noter que les administrateurs sont élus sans limite temporelle, mais peuvent faire l'objet d'une contestation, d'une suspension ou d'une destitution selon des procédures particulières. Voir : Wikipédia (s. d.). Wikipédia:Administrateur. Repéré à https://fr.wikipedia.org/wiki/Wikip\%C3\%A9dia:Administrateur (consulté le 11 janvier 2014).
} 
believe in rough consensus and running code ", "Nous croyons à un consensus approximatif et à un code fonctionnel », tel est le credo adopté par cette organisation œuvrant pour la normalisation des standards de l'Internet qui tente ainsi de concilier une recherche de l'efficacité avec un certain niveau d'accord entre les participants. En certaines circonstances, les wikipédiens peuvent également recourir au vote lors d'une procédure dite de " prise de décision ». Toutefois, ce dispositif n'a pas bonne réputation du fait des divisions communautaires qu'il induit et de son caractère jugé trop bureaucratique (Jemielniak, 2014; Langlais, 2014). La procédure a ainsi graduellement été remplacée par le sondage, un système de vote plus souple.

L'obtention du consensus réglementaire repose sur la transparence, la co-écriture et la discussion collective. La spécificité de la technique d'édition wiki autorise un accord par le jeu des modifications successives de la page consacrée à la règle. Son processus d'écriture est donc le même que celui des articles encyclopédiques. Les documents réglementaires wikipédiens obéissent aux principes d'évolutivité et de distribution égalitaire du pouvoir scriptural sans distinction de niveau d'intégration à la communauté. Toutefois, il est clairement spécifié que l'écriture des règles doit s'accompagner d'un dialogue intracommunautaire dans les pages de discussions associées.

\section{Problématique de la recherche}

Les valeurs égalitaires et démocratiques imprègnent la culture de Wikipédia et la rhétorique à son sujet (Jemielniak, 2016 ; Sahut, 2015). En s'appuyant sur la conception de la démocratie développée par Arend Lijphart, Langlais (2014) a souligné que la construction des règles wikipédiennes résulte d'une délibération rationnelle ouverte et d'un consensus communautaire obtenu par la négociation: "le débat public n'est jamais capté par une petite minorité spécialisée » (p. 33). En ce sens, les espaces de discussion consacrés au système normatif ont été assimilés aux arènes participatives mises en place pour gérer collectivement les biens communs naturels (Viégas, Wattenberg et McKeon, 2007). Conformément au $3^{\mathrm{e}}$ principe d'Ostrom, la possibilité offerte à tous 
La gouvernance de Wikipédia :

élaboration de règles et théorie d'Ostrom

de participer à l'élaboration des règles favoriserait la cohésion de la communauté et la confiance mutuelle. Selon Konieczny (2009), l'ouverture et la transparence des débats permettent de limiter la constitution d'un pouvoir oligarchique, notamment celui des administrateurs. Pourtant, des études montrent l'émergence d'une élite wikipédienne qui exerce une forte influence sur les prises de décision communautaires. Canivenc (2009) met en valeur l'emprise des contributeurs expérimentés qui ont réussi à imposer de nouvelles règles dans la Wikipédia francophone entre 2004 et 2007 , et ce, malgré les résistances d'autres participants. L'étude des désignations des arbitres chargés de gérer les conflits intracommunautaires a témoigné de la surreprésentation nette des administrateurs à la fois comme arbitres et comme participants à ces élections (Jacquemin, 2011). Ce fonctionnement apparemment oligarchique de la gouvernance wikipédienne pourrait apparaître comme une contradiction avec l'esprit égalitaire caractérisant le projet initial. À partir de l'étude de l'activité des contributeurs de 683 wikis de grande taille, Shaw et Hill (2014) ont démontré que leur développement obéissait à la loi d'airain formulée par Robert Michels en 1911. Ils ont ainsi observé la formation d'une élite composée des membres les plus anciens de la communauté et une transformation oligarchique de ces projets éditoriaux. Ces élites y exercent un monopole sur les mécanismes du pouvoir et développent des intérêts propres qu'ils imposent à l'ensemble des participants.

L'étude de la genèse et de l'évolution des règles suppose également d'être attentif aux relations entre les Wikipédia francophone et anglophone. Selon Langlais (2014), l'encyclopédie en langue française s'est rapidement émancipée de la tutelle de son aînée pour développer une gouvernance autonome. Dès 2002, elle a mis en place des normes et des outils de régulation spécifiques. L'étude de Pentzold (2011) amène à nuancer cette affirmation. L'analyse des discussions entre wikipédiens témoigne de l'existence d'un leadership de l'encyclopédie en langue anglaise, dont les normes influent sur celles des autres communautés. Cela nous conduit à nous pencher plus précisément sur les éventuelles influences de la Wikipédia anglophone sur la construction de règles au sein de l'encyclopédie 
en langue française. De même, comme nous l'avons noté plus haut, des acteurs comme Wales et la Wikimedia Foundation sont susceptibles d'intervenir dans ce processus. L'histoire de l'encyclopédie francophone restant à faire, c'est là un sujet qui a été très peu étudié.

Ainsi, on discerne bien qu'il n'y a pas d'unanimité sur la nature "politique " de la gouvernance wikipédienne et sur les facteurs influençant l'élaboration de sa structure réglementaire. Dans cet article, nous nous penchons plus particulièrement sur le fonctionnement du système législatif destiné à construire l'appareil normatif régissant l'encyclopédie. Selon le 3e principe d'Ostrom, la participation de tous à la construction des règles est une condition nécessaire à la gouvernance efficace d'un bien commun. Nous avons donc entrepris une étude quantitative permettant de cerner la proportion de wikipédiens ayant participé aux rédactions et aux discussions à propos de deux règles liées à la citation des sources et au référencement des contenus. De même, nous avons cherché à identifier le profil de ces participants (niveau de participation à l'encyclopédie, rôle formel). Afin de compléter cette étude quantitative, nous nous sommes penché sur le rôle de Wales et de la Wikimedia Foundation dans ce processus. Nous nous livrons également à une approche qualitative des mécanismes décisionnels de Wikipédia. II s'agit ici d'analyser le fonctionnement des arènes participatives - pour reprendre un terme issu de la théorie d'Ostrom - de Wikipédia et les modalités communicationnelles susceptibles d'avoir un rôle décisif dans le processus d'élaboration des deux règles étudiées.

\section{Considérations méthodologiques}

Les données ont été recueillies en nous appuyant sur la mémoire éditoriale de Wikipédia. Le dispositif wiki conserve l'ensemble des versions des textes successivement rédigés associés aux noms - le plus souvent un pseudonyme - des contributeurs qui les ont modifiés, de même que la totalité des discussions qui les ont accompagnées.

Nous avons, dans un premier temps, eu recours à une 
La gouvernance de Wikipédia : élaboration de règles et théorie d'Ostrom

approche quantitative portant sur les modifications de deux textes réglementaires, Citez vos sources et Vérifiabilité, dont le rôle s'est avéré crucial dans le modèle éditorial de Wikipédia (Sahut, 2014). Nous avons recensé les changements significatifs de ces deux règles ayant lieu entre leur création (2004 pour la première, 2006 pour la seconde) et la fin de l'année 2013. Ont ainsi été identifiées 134 modifications de contenu ${ }^{4}$ pour Citez vos sources et 105 pour Vérifiabilité. Nous avons alors procédé au comptage du nombre de rédacteurs ayant participé à ces modifications. Ces données ont été croisées avec celles offertes par le Wikiscan ${ }^{5}$ et Wikistats ${ }^{6}$, outils statistiques qui recensent le nombre total de contributeurs ainsi que le nombre de contributions effectuées par chaque wikipédien. Ce recensement a été effectué en 2014, les indicateurs ainsi retenus reflétant l'activité rédactionnelle globale des différents contributeurs durant la période d'étude du corpus, soit 2004-2013. De même, nous avons eu recours à la liste des administrateurs de l'encyclopédie pour dénombrer ceux qui avaient été impliqués dans la rédaction des deux textes normatifs en vérifiant qu'ils avaient bien ce statut lorsqu'ils sont intervenus sur les pages ${ }^{7}$. Une démarche similaire a été appliquée aux pages de discussion associées à Citez vos sources et à Vérifiabilité, l'objectif étant également de saisir le profil des participants à ces échanges délibératifs.

Afin d'avoir une vision plus globale des différents acteurs pouvant être impliqués dans la genèse des règles étudiées et leurs évolutions, nous avons, dans un deuxième temps, réalisé un entretien semi-directif avec Florence Devouard, l'une des trois premiers administrateurs de la Wikipédia francophone et personnalité très impliquée dans la Wikimedia Foundation, dont elle est devenue vice-présidente en 2004, puis présidente de 2006

\footnotetext{
${ }^{4}$ Nous n'avons pas pris en compte les changements portant sur la forme (orthographe, syntaxe, ajout de liens internes, reformulation sans changement de sens, mise en page, etc.) ainsi que les nombreux vandalismes (grossièretés, phrases n'ayant pas de sens, etc.) et leur révocation.

${ }^{5}$ Disponible à : https://fr.wikiscan.org/.

${ }_{7}^{6}$ Disponible à : https://stats.wikimedia.org/EN/TablesWikipediaFR.htm.

${ }^{7}$ Wikipédia. Wikipédia:Liste des administrateurs. Repéré à https://fr.wikipedia.org/w/index.php?title=Wikip \%C3 \%A9dia:Liste_des_administrateurs\& oldid=845641 (consulté le 15 décembre 2014).
} 
à 2008, succédant à Wales.

Nous n'évoquerons pas ici dans le détail les différents arguments développés dans le cadre de ces échanges autour des règles (voir Sahut, 2014, 2016). En revanche, nous avons été attentif à la nature du dispositif communicationnel, analyse que nous mobiliserons pour mettre en perspective les résultats.

\section{Résultats}

\section{1 Étude quantitative sur les rédacteurs des pages de règles}

Les participants à la rédaction des deux règles étudiées constituent une minorité très restreinte des contributeurs à l'encyclopédie (Tableau 1). Dans les deux cas étudiés, ils sont inférieurs à $0,1 \%$ des contributeurs enregistrés ayant effectué au moins dix modifications dans l'encyclopédie. II est possible d'admettre des critères plus restrictifs de définition d'appartenance communautaire (ici le fait d'avoir effectué plus de 1000 contributions, qui témoigne d'un fort niveau d'engagement dans la communauté); le pourcentage des rédacteurs se situe alors autour des $1 \%$ (1,52\% pour Citez vos sources et $0,92 \%$ pour Vérifiabilité). 
La gouvernance de Wikipédia :

élaboration de règles et théorie d'Ostrom

Tableau 1. Proportion des wikipédiens rédacteurs des règles
de référencement

\begin{tabular}{|l|c|c|c|}
\cline { 2 - 4 } \multicolumn{1}{c|}{} & $\begin{array}{c}\mathbf{N}^{\text {bre }} \text { de } \\
\text { rédacteurs }\end{array}$ & $\begin{array}{c}\% \text { par rapport } \\
\text { au }^{\text {bre }} \text { total de } \\
\text { contributeurs }^{8}\end{array}$ & $\begin{array}{c}\% \text { par rapport } \\
\text { aux contributeurs } \\
\mathbf{>} 1000 \\
\text { contributions }^{9}\end{array}$ \\
\hline $\begin{array}{l}\text { Citez vos } \\
\text { sources }\end{array}$ & 69 & $0,07 \%$ & $1,52 \%$ \\
\hline Vérifiabilité & 42 & $0,04 \%$ & $0,92 \%$ \\
\hline
\end{tabular}

Les administrateurs forment $40 \%$ des rédacteurs des règles (Tableau 2). Si nous rapportons ce chiffre à la proportion d'administrateurs au sein de la population wikipédienne (autour de $1 \%$ des contributeurs actifs), nous constatons qu'ils sont très largement surreprésentés dans le cadre de ce travail législatif. Toutefois, si on considère l'ensemble des administrateurs de l'encyclopédie, il s'avère que seule une minorité d'entre eux se sont attelés à cette activité rédactionnelle (8 à $13 \%)^{10}$. Par ailleurs, ils n'ont pas l'exclusivité de l'écriture réglementaire. Ils sont en effet responsables de $31 \%$ des modifications de Citez vos sources et de $40 \%$ pour Vérifiabilité.

\footnotetext{
${ }^{8}$ Pour les calculs statistiques, le contributeur est défini comme quelqu'un qui a un compte dans l'encyclopédie et qui a effectué au moins dix modifications dans l'encyclopédie depuis la création de ce compte (Mediawiki Analytics/Metric definitions https://www.mediawiki.org/wiki/Analytics/Metric_definitions [consulté le 23 février 2014]). Nous nous sommes fondé sur le nombre de contributeurs qui répondent à cette définition en décembre 2013, soit 94317 (statistiques fournies par la Wikimedia Foundation, https://stats.wikimedia.org/FR/TablesWikipediaFR.htm [consulté le 12 février 2014]).

9 Wikimedia Foundation (2015, juin). Statistiques Wikipédia français. Repéré à https://stats.wikimedia.org/FR/TablesWikipediaFR.htm [consulté le 10 juillet 2015]). II faut considérer, ici, le pourcentage donné comme un ordre de grandeur puisque ce nombre a évolué durant la période considérée.

${ }^{10}$ Rappelons ici aussi que ces chiffres ont une valeur relative puisque le nombre d'administrateurs et leur proportion ont varié au cours du projet. Ils sont considérés comme des ordres de grandeur.
} 
Tableau 2. Part des administrateurs dans la rédaction de la règle

\begin{tabular}{|c|c|c|c|}
\hline & $\begin{array}{c}\mathrm{N}^{\mathrm{bre}} \\
\text { d'adminis- }^{\text {trateurs }} \\
\text { trateurs } \\
\text { rédacteurs }\end{array}$ & $\begin{array}{c}\% \text { par rapport } \\
\text { au } \mathrm{n}^{\text {bre }} \text { total de } \\
\text { rédacteurs }\end{array}$ & $\begin{array}{c}\% \text { de } \\
\text { modifications } \\
\text { par les } \\
\text { administrateurs }\end{array}$ \\
\hline $\begin{array}{l}\text { Citez vos } \\
\text { sources }\end{array}$ & 28 & $41 \%$ & $31 \%$ \\
\hline Vérifiabilité & 17 & $40 \%$ & $40 \%$ \\
\hline
\end{tabular}

Pour compléter cette approche, nous voulons cerner le profil des rédacteurs selon un autre critère : le nombre de contributions à l'encyclopédie. Le contenu des règles a été principalement modifié par des wikipédiens appartenant au $1 \%$ des membres les plus actifs de la communauté (en quasi-totalité pour Vérifiabilité, en très grande majorité pour Citez vos sources) (Tableaux 3 et 4). Nous avons également observé que près d'un rédacteur sur deux $(48 \%)$ ayant participé à la rédaction de Vérifiabilité a également contribué à celle de Citez vos sources. Ce constat renforce l'idée d'une forte concentration de l'activité normative communautaire. 
La gouvernance de Wikipédia :

élaboration de règles et théorie d'Ostrom

Tableau 3. Profil des rédacteurs des règles selon leur niveau de participation à l'encyclopédie

\begin{tabular}{|l|c|c|c|}
\cline { 2 - 4 } \multicolumn{1}{c|}{} & $\begin{array}{l}\text { Moyenne des } \\
\text { contributions } \\
\text { par rédacteur }\end{array}$ & $\begin{array}{c}\text { \% de } \\
\text { rédacteurs } \\
\text { appartenant } \\
\text { aux } \mathbf{0 , 9} \% \\
\text { wikipédiens } \\
\text { les plus } \\
\text { actifs }\end{array}$ & $\begin{array}{c}\text { 11 } \\
\text { rédacteurs } \\
\text { appartenant } \\
\text { aux } \mathbf{0 , 5} \% \\
\text { wikipédiens } \\
\text { les plus } \\
\text { actifs }\end{array}$ \\
\hline $\begin{array}{l}\text { Citez vos } \\
\text { sources }\end{array}$ & 17174 & $88 \%$ & $77 \%$ \\
\hline Vérifiabilité & 18799 & $95 \%$ & $85 \%$ \\
\hline
\end{tabular}

Tableau 4. Pourcentage de modifications des règles selon le niveau de participation des contributeurs à l'encyclopédie

\begin{tabular}{|l|c|c|}
\cline { 2 - 3 } \multicolumn{1}{c|}{} & $\begin{array}{c}\text { \% de modifications } \\
\text { par rédacteurs } \\
\text { appartenant aux } \\
\mathbf{0 , 9} \% \text { wikipédiens } \\
\text { les plus actifs }\end{array}$ & $\begin{array}{c}\% \text { de modifications } \\
\text { par rédacteurs } \\
\text { appartenant aux } \\
\mathbf{0 , 5} \% \text { wikipédiens les } \\
\text { plus actifs }\end{array}$ \\
\hline $\begin{array}{l}\text { Citez vos } \\
\text { sources }\end{array}$ & $85 \%$ & $72 \%$ \\
\hline Vérifiabilité & $98 \%$ & $90 \%$ \\
\hline
\end{tabular}

\footnotetext{
${ }^{11}$ Nous nous sommes fondé sur les statistiques fournies par la Wikimedia Foundation (Wikimedia Foundation [2015, juin]. Distribution of article edits over wikipedians. Repéré à https://stats.wikimedia.org/FR/TablesWikipediaFR.htm [consulté le 5 juin 2014]). Cette catégorie rassemble les contributeurs ayant effectué plus de 1000 éditions dans l'espace encyclopédique.

${ }^{12}$ Cette catégorie regroupe les contributeurs ayant effectué plus de 3162 éditions dans l'espace encyclopédique (Wikimedia Foundation [2014, juin]. Distribution of article edits over wikipedians. Repéré à https://stats.wikimedia.org/FR/TablesWikipediaFR.htm [consulté le 5 juin 2014]).
} 
Nous avons mis en œuvre le même type d'analyse pour les pages de discussions associées.

\section{2 Étude quantitative sur les participants aux discussions}

Les proportions de participants aux discussions sur les règles sont également très réduites: $0,1 \%$ pour la totalité des contributeurs enregistrés ayant effectué au moins dix modifications dans les articles de Wikipédia, et autour de $2 \%$ si on adopte une définition encore plus restrictive de la communauté (Tableau 5$)^{13}$.

Tableau 5. Nombre et pourcentage de participants aux discussions sur le référencement

\begin{tabular}{|l|c|c|c|}
\cline { 2 - 4 } \multicolumn{1}{c|}{} & $\begin{array}{c}\text { N } \\
\text { participants } \\
\text { aux } \\
\text { discussions }\end{array}$ & $\begin{array}{c}\text { be par rapport } \\
\text { au } \mathbf{n}^{\text {bre }} \text { total de } \\
\text { contributeurs }\end{array}$ & $\begin{array}{c}\% \text { par rapport } \\
\text { au } \mathbf{n}^{\text {bre }} \text { de } \\
\text { wikipédiens }> \\
\mathbf{1 0 0 0} \\
\text { contributions }\end{array}$ \\
\hline $\begin{array}{l}\text { Citez vos } \\
\text { sources }\end{array}$ & 113 & $0,1 \%$ & $2,5 \%$ \\
\hline Vérifiabilité & 89 & $0,09 \%$ & $2 \%$ \\
\hline
\end{tabular}

Nous retrouvons une surreprésentation des administrateurs dans les discussions autour des règles, même si les pourcentages de participation s'avèrent légèrement inférieurs (de $10 \%$ environ) par rapport à ceux ayant trait à la rédaction des règles (Tableau 6).

\footnotetext{
${ }^{13}$ Nous reprenons le critère d'un nombre de contributions supérieur à 1000.
} 
La gouvernance de Wikipédia :

élaboration de règles et théorie d'Ostrom

Tableau 6. Nombre et pourcentage d'administrateurs participant aux discussions sur le référencement.

\begin{tabular}{|l|c|c|c|}
\cline { 2 - 4 } \multicolumn{1}{c|}{} & $\begin{array}{c}\mathbf{N}^{\text {bre }} \\
\text { d'administrateurs } \\
\text { discutant }\end{array}$ & $\begin{array}{c}\text { \% par } \\
\text { rapport au } \\
\mathbf{n}^{\text {bre }} \text { total de } \\
\text { discutant }\end{array}$ & $\begin{array}{c}\text { \% de messages } \\
\text { par les } \\
\text { administrateurs }\end{array}$ \\
\hline $\begin{array}{l}\text { Citez vos } \\
\text { sources }\end{array}$ & 34 & $30 \%$ & $30 \%$ \\
\hline Vérifiabilité & 25 & $28 \%$ & $21 \%$ \\
\hline
\end{tabular}

En s'appuyant sur le critère du nombre de contributions, nous relevons que les discussions sont très majoritairement alimentées par des contributeurs prolifiques de l'encyclopédie (Tableau 7). Le nombre moyen de contributions par discutant est à peine moins élevé que celui des rédacteurs : 15000 pour Citez vos sources, 14567 pour Vérifiabilité (Tableau 8). Nous notons enfin qu'un tiers des participants aux discussions sur la règle Vérifiabilité ont aussi pris part à celles concernant Citez vos Sources.

Tableau 7. Modifications apportées aux règles selon le niveau de participation à l'encyclopédie

\begin{tabular}{|l|c|c|}
\cline { 2 - 3 } \multicolumn{1}{c|}{} & $\begin{array}{c}\text { \% de messages } \\
\text { par discutant } \\
\text { appartenant aux } \\
\mathbf{0 , 9} \% \text { wikipédiens } \\
\text { les plus actifs }\end{array}$ & $\begin{array}{c}\% \text { de messages par } \\
\text { discutant appartenant } \\
\text { aux 0,5 \% wikipédiens } \\
\text { les plus actifs }\end{array}$ \\
\hline $\begin{array}{l}\text { Citez vos } \\
\text { sources }\end{array}$ & $80 \%$ & $70 \%$ \\
\hline Vérifiabilité & $97 \%$ & $82 \%$ \\
\hline
\end{tabular}


Tableau 8. Profil des rédacteurs des règles selon le niveau de participation à l'encyclopédie

\begin{tabular}{|c|c|c|c|}
\hline & $\begin{array}{l}\text { Moyenne des } \\
\text { contributions } \\
\text { par discutant }\end{array}$ & $\begin{array}{c}\% \text { de discutant } \\
\text { appartenant } \\
\text { aux } 0,9 \% \\
\text { wikipédiens } \\
\text { les plus } \\
\text { actifs }^{14}\end{array}$ & $\begin{array}{c}\% \text { de } \\
\text { discutant } \\
\text { appartenant } \\
\text { aux } 0,5 \% \\
\text { wikipédiens } \\
\text { les plus } \\
\text { actifs }^{15}\end{array}$ \\
\hline $\begin{array}{l}\text { Citez vos } \\
\text { sources }\end{array}$ & 15000 & $65 \%$ & $55 \%$ \\
\hline Vérifiabilité & 14567 & $82 \%$ & $73 \%$ \\
\hline
\end{tabular}

\subsection{Les acteurs à l'échelle internationale}

Afin d'aboutir à une vision plus complète de ce processus de construction des règles, nous nous sommes intéressé aux influences des autres acteurs de la gouvernance wikipédienne que sont Wales et la Wikimédia Foundation, ainsi qu'au rôle de la Wikipédia anglophone qui fait figure de projet encyclopédique collaboratif séminal.

Cette influence s'exerce de manière directe. Elle concerne tout d'abord la création de la règle de Vérifiabilité, rédigée pour la première fois le 5 janvier 2006 par un administrateur dénommé Ske ${ }^{16}$. Celle-ci se fait en l'absence de délibérations

\footnotetext{
${ }^{14}$ Nous nous sommes fondé sur les statistiques fournies par la Wikimedia Foundation (Wikimedia Foundation [2015, juin]. Distribution of article edits over wikipedians. Repéré à https://stats.wikimedia.org/FR/TablesWikipediaFR.htm [consulté le 5 juin 2015]). Cette catégorie rassemble les contributeurs ayant effectué plus de 1000 éditions dans l'espace encyclopédique

${ }^{15}$ Cette catégorie regroupe les contributeurs ayant effectué plus de 3162 éditions dans l'espace encyclopédique. (Wikimedia Foundation [2015, juin]. Distribution of article edits over wikipedians. Repéré à https://stats.wikimedia.org/FR/TablesWikipediaFR.htm [consulté le 5 juin 2015]).

16 Ske occupe cette fonction depuis 2004 (Wikipédia [2004, 16 septembre]. Wikipédia:Liste des administrateurs. Repéré à

https://fr.wikipedia.org/w/index.php?title=Wikip \%C3 \%A9dia:Liste_des_administrateurs\& oldid=845641\#Candidature_de_Ske [consulté le 2 avril 2018]).
} 
La gouvernance de Wikipédia : élaboration de règles et théorie d'Ostrom

prédécisionnelles dans les espaces de discussion wikipédiens étudiés ${ }^{17}$. Pour rédiger ce texte réglementaire, Ske s'inspire tout d'abord largement de la version de cette règle en vigueur dans la Wikipédia anglophone, dont il sera un contributeur régulier à partir de 2007. Cette décision se comprend si on examine le contexte d'évolution du projet encyclopédique qui connaît une grave crise durant l'hiver 2005. John Seigenthaler, un journaliste américain réputé, menace de porter plainte contre la Wikipédia anglophone après avoir découvert des accusations injustifiées contre sa personne proférées dans l'article qui lui est consacré. Cette affaire médiatisée nuit à la réputation naissante de l'encyclopédie collaborative et semble faire peser une menace juridique sur son existence (Sahut, 2016). Wales et la Wikimedia Foundation réagissent en instaurant une règle spécifique portant sur les biographies de personnes vivantes dans la Wikipédia anglophone (Lih, 2009). Les informations ne peuvent désormais être acceptées que si elles sont clairement rattachées à des sources fiables par une référence bibliographique. Plus encore, cette affaire provoque une prise de conscience de la perméabilité de Wikipédia face à l'introduction d'informations inexactes. La citation des sources paraît être la solution adéquate face à ce type de problème. En tant que présidente du conseil d'administration de la Wikimedia Foundation de 2006 à 2008, Florence Devouard dit avoir beaucoup œuvré afin que les communautés des différentes versions linguistiques de l'encyclopédie adoptent cette règle. Ces facteurs externes à la Wikipédia francophone semblent donc expliquer la création de la première version Vérifiabilité au début de l'année 2006 sans que des débats préalables n'aient eu lieu.

Nous avons également repéré une influence plus diffuse de la Wikipédia anglophone. En 2006-2007, les règles relatives au référencement sont contestées par une partie des wikipédiens francophones. Entre autres arguments, les partisans mobilisent les textes réglementaires de la Wikipédia en langue anglaise et les prises de position de Wales favorables à son instauration. La force du lien avec le "projet mère » est convoquée pour repousser les

\footnotetext{
${ }^{17}$ Nous avons également systématiquement dépouillé les discussions sur le Bistro, qui joue le rôle de forum central de la communauté.
} 
contestations des règles ou certaines modifications.

\section{Discussion et analyses complémentaires}

\subsection{L'activité d'une minorité, une oligarchie wikipédienne?}

On savait que l'exercice du pouvoir scriptural pour l'écriture des articles encyclopédiques de Wikipédia était très concentré. Cette concentration est encore plus marquée pour la rédaction et la discussion des règles, puisque seule une ultra-minorité des wikipédiens les plus impliqués dans le projet y a participé. Nous mettrons tout d'abord l'accent sur la forte influence des administrateurs dans le processus d'institutionnalisation de Wikipédia. Alors que leur statut officiel les cantonne à un rôle technique et d'application des décisions communautaires, deux d'entre eux sont à l'origine des règles sur le référencement ${ }^{18}$ et d'autres figurent parmi les acteurs centraux de leur rédaction et des discussions à leur sujet. Ils n'ont pas toutefois le monopole du " pouvoir législatif » wikipédien. Ils le partagent en effet avec des contributeurs particulièrement expérimentés. Nos résultats sont donc convergents avec ceux de Konieczny (2009) portant sur la Wikipédia anglophone.

II apparaît que la grande majorité des contributeurs ont préféré participer à la rédaction des articles encyclopédiques plutôt qu'aux discussions et aux décisions politiques ou n'aient pas été informés des changements réglementaires en cours. Cette désaffection peut être interprétée comme une acceptation silencieuse tout autant que le ressenti d'un manque de légitimité ou d'intérêt pour la gouvernance de Wikipédia. Nous notons également que les tâches de gouvernance étudiées ont été assumées par des volontaires qui se sont investis parallèlement dans les activités rédactionnelles et réglementaires. Nous pourrions dire qu'il n'y a pas, dans Wikipédia, de " classe politique » coupée du terrain.

18 Anthere alias Florence Devouard pour Citez vos sources, Ske pour Vérifiabilité 
La gouvernance de Wikipédia :

élaboration de règles et théorie d'Ostrom

Ce constat sur la concentration du pouvoir législatif dans l'entreprise wikipédienne nous amène à réexaminer le premier principe d'Ostrom, à savoir l'existence de règles claires définissant l'appartenance communautaire. Les études initiales d'Ostrom ont porté sur des biens communs matériels situés d'un point de vue géographique. L'appartenance communautaire se décide sur la base d'une contribution à une activité économique commune et régulière ancrée dans un territoire. Ces caractéristiques favorisent un certain degré d'homogénéité culturelle ainsi qu'une appréhension largement partagée de la gouvernance du bien commun, notamment quand il s'agit de faire évoluer les règles (Basurto et Ostrom, 2011).

Par rapport à ce type de communs, le projet wikipédien présente un certain nombre de traits spécifiques. II est à la fois déterritorialisé, ouvert à la contribution de n'importe quels volontaires, qui, dans leur grande majorité, y participent sur leur temps libre. II existe de fait une forte hétérogénéité des niveaux d'implication dans le projet encyclopédique et l'on peut distinguer des contributeurs débutants - recrutés parmi les lecteurs de l'encyclopédie -, occasionnels et réguliers (Bryant, Forte et Bruckman, 2005). Cette "structure en oignon ", que l'on retrouve dans les communautés de logiciel libre (Broca, 2013), a des conséquences sur le plan de la gouvernance. La participation aux discussions sur les règles, voire à leur rédaction, est une activité qui a un coût relativement élevé. II faut non seulement être capable de contribuer à l'encyclopédie (maîtrise de l'interface d'écriture, compétences rédactionnelles...) mais, de surcroît, avoir des connaissances précises sur les enjeux et les problématiques du projet, sur les normes sociales et communicationnelles en vigueur. S'exprimer au sein de la communauté sur le devenir de Wikipédia suppose également d'éprouver un sentiment de légitimité qui ne s'acquiert que par une participation inscrite dans la durée. On comprend ainsi que, malgré une ouverture de principe, cette activité législative ait été prise en main par une minorité fortement engagée.

\subsection{Rationalité et normes collectives}


Pour autant, peut-on parler, comme l'ont fait Shaw et Hill (2014), d'une loi d'airain qui serait à l'œuvre dans la gouvernance de ce commun de la connaissance ? Cela nous paraît excessif. II nous semble difficile de voir dans les processus délibératifs et décisionnels étudiés, la manifestation d'une rationalité caractérisant un homo œconomicus qui adopterait un comportement visant à maximiser ses bénéfices individuels. L'analyse qualitative des discussions menées dans notre recherche doctorale montre que les interventions ne reflètent visiblement pas la recherche d'un intérêt personnel (Sahut, 2015). Le noyau central de la communauté qui a pris en charge la gouvernance de l'encyclopédie formule ce qu'il pense être les meilleures solutions à adopter pour le projet collectif, même s'il arrive que les représentations de celui-ci soient divergentes. II a nourri sa réflexivité à partir d'une analyse critique de l'œuvre en cours de réalisation. Le choix d'adopter des règles relatives à la citation des sources paraît ici rationnel, au sens où il résulte d'une réflexion durant laquelle de nombreuses solutions ont été envisagées et évaluées. Les abondants débats ont notamment porté sur les avantages et les inconvénients de la citation, la nature des sources pouvant être citées, les cas où la citation revêt un caractère obligatoire ou facultatif (Sahut, 2015). Comme dans les communautés étudiées par Ostrom (2010), les wikipédiens impliqués dans le processus décisionnel ont évalué les coûts et les bénéfices de la citation pour le devenir de Wikipédia.

Il est cependant nécessaire de pointer à nouveau une différence avec les biens communs naturels. Leur gouvernance s'appuie sur un capital de connaissances et de valeurs issu de la tradition, qui est mobilisé afin de gérer le système mis en place (Basurto et Ostrom, 2011). À ces débuts, Wikipédia est quant à lui un projet novateur qui ne bénéficie pas de tels acquis. La nature même des buts poursuivis, les modalités à mettre en œuvre pour les atteindre et l'identité collective ont été définies progressivement dans les premières années du projet. Entre 2004 et 2008, les débats sont l'occasion d'affirmer l'importance d'un objectif essentiel de l'entreprise commune : la fiabilité des connaissances exposées. Le référencement des contenus est notamment considéré comme un moyen de rattacher les énoncés encyclopédiques à des sources 
La gouvernance de Wikipédia :

élaboration de règles et théorie d'Ostrom

dotées d'une certaine autorité (Langlais, 2015; Sahut, 2014, 2015). Cette pratique est devenue par la suite un marqueur identitaire du projet wikipédien. Cette analyse rejoint les études montrant que les normes sociales et l'identité collective des communautés en ligne se construisent au cours des interactions entre les participants (Dang Nguyen, Dejean et Jullien, 2018; Zhou, 2011).

Nous avons également observé l'influence décisive de Wales, de la Wikimedia Foundation et, plus globalement, de la Wikipédia anglophone sur l'instauration des règles étudiées. Les interventions des deux premiers acteurs lors de l'affaire Seigenthaler relève d'un processus adaptatif conscient, mécanisme qui a été fréquemment repéré dans les communautés gérant des ressources naturelles (Basurto et Ostrom, 2011). Nos analyses tendent à montrer qu'au moins dans les premières années d'existence de l'encyclopédie francophone, la Wikipédia anglophone est considérée comme un modèle à imiter en raison de son dynamisme et de son efficience. Nos résultats vont donc dans le sens de celles de Pentzold (2011), qui a mis en évidence son leadership sur les autres versions linguistiques. Ce phénomène d'influence se retrouve dans des systèmes de gouvernances étudiés par Ostrom (2010). Afin de réduire l'incertitude inévitablement associée à tout changement de règles, certaines communautés optent pour des règles qui ont fait leurs preuves dans des collectifs similaires.

En ce sens, l'imitation de la Wikipédia anglophone par la communauté francophone peut être considérée comme la manifestation d'une rationalité collective. Comme chez Ostrom qui s'est elle-même inspirée d'Herbert Simon (Chanteau et Labrousse, 2013), il s'agit d'une rationalité limitée et située. Les wikipédiens ont privilégié des solutions en adéquation avec le système écologique et local dans lequel ils évoluent. Menant leur réflexion collective de manière endogène, ils n'ont pas, à quelques exceptions près, convoqué de travaux scientifiques ou de textes réflexifs sur la citation des sources ni d'ailleurs sur les notions de fiabilité et de crédibilité, thèmes qui ont été au centre de leurs débats. Ils ont privilégié une argumentation reposant sur une connaissance distribuée localement entre les participants aux 
projets wikipédiens.

5.3 Une arène soutenue par un dispositif communicationnel adapté

Les conditions communicationnelles dans lesquelles s'opèrent les échanges entre wikipédiens ont pu favoriser cet exercice de rationalité collective. À la suite de Viégas, Wattenberg, Kriss et Van Ham (2007), nous évoquerons tout d'abord la complémentarité entre les différents espaces textuels de Wikipédia. L'espace réglementaire est ainsi articulé à l'espace encyclopédique et aux espaces de discussions (Figure 1).

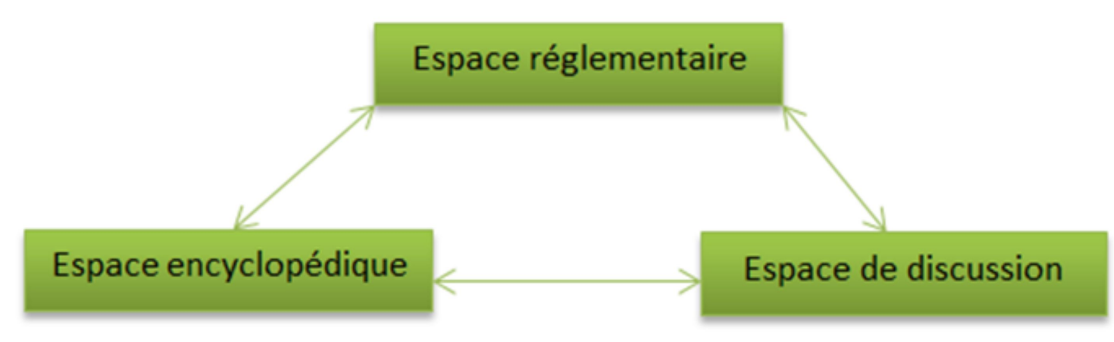

Figure 1. Articulation entre les différents espaces wikipédiens

En ce sens, Viégas, Wattenberg, Kriss et Van Ham (2007) ont souligné qu'un nombre non négligeable de messages présents sur les pages de discussion associées aux articles comportaient des références explicites aux règles de l'encyclopédie. De manière complémentaire, nous avons observé que nombre de discussions sur les règles prennent appui sur l'expérience contributrice. II existe un va-et-vient constant entre l'appréciation de cas rédactionnels jugés problématiques ou exemplaires et un niveau plus abstrait, où la discussion se déplace vers des considérations plus générales concernant le référencement, voire la politique éditoriale globale de l'encyclopédie. La réflexion wikipédienne se nourrit d'un double mouvement inductif et déductif qui se matérialise par des liens hypertextes. Lors de discussions sur les règles du référencement, ceux qui ont pointé vers l'espace encyclopédique ont donc eu plusieurs fonctions : 
La gouvernance de Wikipédia :

élaboration de règles et théorie d'Ostrom

- Une fonction d'alerte : le wikipédien attire l'attention des autres contributeurs sur l'état ou les évolutions d'un article

- Une fonction de clarification cognitive: le contributeur donne un exemple situé dans l'encyclopédie ou une autre discussion dans le but de mieux se faire comprendre. II arrive aussi que les articles de l'encyclopédie soient cités simplement pour leur valeur référentielle, la notion développée dans un article évitant d'insérer une définition dans la discussion.

- Une fonction argumentative: l'exemple donné vient en appui d'un argument. II peut ainsi témoigner d'une pratique qui devrait être imitée. II joue alors le rôle de modèle. II arrive à l'inverse qu'il fasse figure de repoussoir.

Ces relations entre l'espace d'élaboration réglementaire et l'espace encyclopédique favorisent la prise de conscience collective des problèmes en lien avec la citation des sources, l'appropriation des règles par la communauté et l'examen de leur adéquation à la pratique rédactionnelle. Nous retrouvons ici le second principe d'Ostrom qui met en exergue la recherche de la congruence entre les évolutions des règles et les conditions spécifiques de leurs applications. En effet, la perception de l'incidence des règles adoptées sur les modes collectifs d'action constitue un élément clé de gouvernance. Tout changement de règles ne conduit pas nécessairement à une amélioration du système. Certaines évolutions peuvent provoquer des déséquilibres intracommunautaires et une désorganisation de l'action collective (Basurto et Ostrom, 2011). Dans Wikipédia, les contributeurs ont pu suivre les effets de l'imposition de la citation des sources sur les modes d'écriture des articles. Ils ont aussi échangé des arguments sur le sujet en s'appuyant sur des exemples concrets issus des articles encyclopédiques et se sont efforcés de recenser les problèmes induits par cette pratique (Sahut, 2015). L'attention portée aux conditions concrètes d'écriture les a amenés à modérer les exigences liées à la citation des sources. Dans les débats étudiés, beaucoup de wikipédiens se sont montrés conscients des coûts cognitifs et temporels induits par cette pratique et ont plaidé pour une adoption souple et 
pragmatique de ces règles. Ils ont ainsi refusé d'adopter des critères trop restrictifs quant à la qualité des sources citées pour ne pas entraver le dynamisme éditorial de l'encyclopédie. Comme dans les communautés gérant des ressources naturelles (Ostrom, 2010), les participants au débat ont été collectivement capables d'identifier les bénéfices escomptés de la citation ainsi que les coûts attachés à cette pratique pour soutenir ou contester un changement de ces règles.

\subsection{L'importance de la chronologie du projet}

Le facteur chronologique a une forte incidence sur l'appréhension communautaire des règles. Pour Citez vos Sources et Vérifiabilité, les principales discussions se situent entre 2006 et 2009. Jusqu'en 2007, c'est surtout le principe même de citation des sources qui fait l'objet de désaccords. De manière progressive, celui-ci est accepté et les discussions se déplacent vers des thèmes connexes : quand est-il nécessaire de citer une source ? Quel type de sources citer ? (Sahut, 2015). Quand, en 2012, un contributeur argumente pour abolir la règle Citez vos sources, il se heurte à un refus ferme et unanime de neuf autres wikipédiens. Cette règle a acquis une légitimité qui la rend résistante à la contestation. Elle fait désormais partie intégrante de l'identité wikipédienne et du répertoire partagé de la communauté.

Ainsi, la logique qui prédomine actuellement en matière de citation des sources est celle du consentement et de l'apprentissage plutôt que celle de la recherche du consensus autour des règles. L'appropriation de cette pratique est considérée comme nécessaire à une véritable intégration dans la communauté. En ce sens, on discerne des initiatives communautaires visant à favoriser cet apprentissage. En 2016, un $\mathrm{MOOC}^{19}$ conçu par des wikipédiens volontaires avec le soutien de l'association Wikimédia France a été lancé afin de "recruter » de nouveaux contributeurs. Deux vidéos issus du WikiMOOC ont été incluses dans la page Citez vos sources. Ces ressources

\footnotetext{
${ }^{19}$ Les Massive Online Open Courses, ou "formations en ligne ouvertes à tous », sont des formations à distance généralement gratuites reposant sur l'usage de ressources numériques.
} 
La gouvernance de Wikipédia :

élaboration de règles et théorie d'Ostrom

didactiques explicitent les fonctions du "sourçage " au sein du modèle éditorial wikipédien et s'efforcent de clarifier quelles sont les sources jugées acceptables par la communauté. Celle-ci a donc conscience de la difficulté d'appropriation de la citation des sources par les quidams. II existe donc une volonté de remédier à cette augmentation des coûts d'entrée communautaires par la mise en œuvre d'une pédagogie ciblée pour les novices potentiellement intéressés par la participation au projet.

De même, le facteur chronologique a également une importance quant à l'influence de la Wikipédia anglophone sur l'encyclopédie francophone. Dans le corpus analysé, nous avons en effet relevé, à partir de la fin des années 2000, plusieurs refus de voir les règles en anglais traduites sans discussions intracommunautaires préalables alors qu'antérieurement, cette pratique n'avait pas soulevé d'oppositions. Nous pourrions donc y voir le signe d'une autonomisation accrue du projet francophone. Un autre exemple va dans ce sens. La volonté de lutter contre le vandalisme a conduit les Wikipédia germanique et anglophone à adopter, à la fin des années 2000, le système des Flagged Revisions (révisions marquées), qui représente une évolution décisive du modèle éditorial. Les nouvelles contributions émanant des contributeurs non inscrits ou des contributeurs inscrits mais n'ayant pas effectué un nombre suffisant d'éditions sont examinées par des wikipédiens expérimentés avant d'être publiées. Dans la communauté francophone, le débat sur les "révisions marquées " a eu lieu en octobre 2009. Un sondage communautaire a été organisé afin que les contributeurs se prononcent sur l'éventuelle adoption du système allemand ou du système anglais. Le premier a été rejeté par $78 \%$ des votants et le deuxième par $56,4 \%{ }^{20}$. Cette émancipation pourrait témoigner d'une certaine maturité du projet francophone à la fin des années 2000. Elle est aussi le signe d'une culture wikipédienne rétive à des formes d'autorité descendante (Sahut, 2016) ainsi que d'une difficulté à centraliser le processus décisionnel dans une communauté portée sur la contestation (Jemielniak, 2016).

${ }^{20}$ Wikipédia (2009). Wikipédia:Sondage/Flagged revisions. Repéré à https://fr.wikipedia.org/wiki/Wikip\%C3\%A9dia:Sondage/Flagged revisions (consulté le 13 décembre 2014). 


\section{Conclusion}

Les échanges autour de l'arsenal réglementaire étudié n'ont impliqué qu'une très faible minorité des participants à l'encyclopédie. Si les finalités de Wikipédia sont, d'un point de vue épistémique, démocratiques (le partage universel du savoir comme horizon), son mode de gouvernance l'est beaucoup moins. Nos conclusions rejoignent ainsi celle de Konieczny (2010), qui a souligné la dimension adhocratique de Wikipédia. Un petit groupe de contributeurs prolifiques et d'administrateurs a pris en charge à la fois les discussions sur les règles étudiées et leur rédaction en agissant dans ce qu'ils pensaient être l'intérêt général du projet. Toutefois, le rapprochement avec les systèmes politiques présente d'importantes limites du fait des spécificités de l'organisation politique de Wikipédia.

On peut en revanche relever de fortes similitudes avec la gouvernance des biens communs théorisée par Ostrom. Certes, nous avons montré que la participation de tous à l'élaboration réglementaire $\left(3^{\mathrm{e}}\right.$ principe) était davantage une virtualité qu'une réalité. Ce constat est notamment dû aux coûts de participation au sein d'un collectif dont les membres ont un niveau d'engagement très hétérogène dans le projet. Néanmoins, l'arène wikipédienne autorise une forme de rationalité partagée qui tient à l'évaluation collective et pragmatique des solutions réglementaires envisagées, réflexion elle-même nourrie par la dimension internationale de cette entreprise. C'est le même processus réflexif qui conduit la communauté à prendre conscience des coûts impliqués par le respect des règles relatives à la citation des sources et à mettre en œuvre une pédagogie spécifique pour y remédier. Ce sont peutêtre là des éléments de la réussite wikipédienne dont d'autres collectifs formés autour de biens communs pourraient s'inspirer.

\section{Références}

Auray, N., Hurault-Plantet, M., Poudat, C. et Jacquemin B. (2009). La négociation des points de vue : une cartographie sociale des conflits et des querelles dans le Wikipédia francophone. Réseaux, 154, 15-50. 
La gouvernance de Wikipédia :

élaboration de règles et théorie d'Ostrom

Barbe, L. (2006). Wikipedia et Agoravox : des nouveaux modèles éditoriaux ?. Dans G. Chartron et E. Broudoux (dir.), Document numérique et société : actes de la conférence DocSoc (p. 5065). Paris. France : ADBS.

Basurto, X. et Ostrom, E. (2011). Crafting Analytical Tools to Study Institutional Change. Journal of Institutional Economics, 7(3), 317-343.

Beaude, B. (2015). De quoi Wikipédia est-elle le lieu?. Dans L. Barbe, L. Merzeau et V. Schafer (dir.), Wikipédia objet scientifique non identifié (p.41-54). Paris, France: Presses universitaires de Paris Ouest.

Benkler, Y. (2009). La Richesse des réseaux : marchés et libertés à l'heure $d u$ partage social. Lyon, France: Presses universitaires de Lyon.

Broca, S. (2013). Utopie du logiciel libre : du bricolage informatique à la réinvention sociale. Neuvy-en-Champagne, France: Le Passager clandestin.

Broudoux, E. (2015). Wikipédia, objet de recherches: entre observations, expérimentations et co-constructions. Dans L. Barbe, L. Merzeau et V. Schafer (dir.), Wikipédia objet scientifique non identifié (p. 55-73). Paris, France: Presses universitaires de Paris Ouest.

Bryant, S. L., Forte, A. et Bruckman A. (2005). Becoming Wikipedian: Transformation of participation in a collaborative online encyclopedia. Dans Proceedings of the 2005 international ACM SIGGROUP conference on Supporting group work (p. 110). New York, NY : Association for Computing Machinery.

Butler, B., Joyce, E. et Pike, J. (2008). Don't look now, but we've created a bureaucracy: The nature and roles of policies and rules in Wikipedia. Dans Proceedings of the SIGCHI Conference on Human Factors in Computing Systems (p. 1101-1110). New York, NY : Association for Computing Machinery.

Canivenc, S. (2009). Autogestion et nouvelles formes organisationnelles dans la société de l'information, de la 
communication et du savoir (Thèse de doctorat). Université Rennes 2, France.

Cardon, D. et Levrel, J. (2009). La vigilance participative. Une interprétation de la gouvernance de Wikipédia. Réseaux, 154, 51-89.

Cardon, D. (2015). Surveiller sans punir. La gouvernance de Wikipédia. Dans L. Barbe, L. Merzeau et V. Schafer (dir.), Wikipédia objet scientifique non identifié (p. 15-39). Paris, France : Presses universitaires de Paris Ouest.

Chanteau, J.-P. et Labrousse, A. (2013). L'institutionnalisme méthodologique d'Elinor Ostrom: quelques enjeux et controverses. Revue de la régulation, 14. Repéré à http://regulation.revues.org/10555

Dang Nguyen, G., Dejean, S. et Jullien, N. (2018). Do open projects create social norms? Journal of Institutional Economics, 14(1), 45-70.

Fallery, B. et Rodhain F. (2013). Gouvernance d'Internet, gouvernance de Wikipédia : l'apport des analyses d'E. Ostrom sur l'action collective auto-organisée. Management \& Avenir, 65(7), 169-188.

Forte, A., Larco, V. et Bruckman, A. (2009). Decentralization in Wikipedia Governance. Journal of Management Information Systems, 26(1), 49-72.

Jacquemin, B. (2011). Autorégulation de rapports sociaux et dispositif dans Wikipédia. Document numérique, 14(3), 57-79

Jemielniak, D. (2014) Common knowledge: An Ethnography of Wikipedia. Stanford, CA : Stanford University Press.

Jemielniak, D. (2016). Wikimedia movement governance: The limits of a-hierarchical organization. Journal of Organizational Change Management, 29(3), 361-378.

Joud, E., Jullien, N. et Le Gall-Ely, M. (2016). Le processus d'acceptation d'un bot : analyse du récit de vie de Salebot. Dans Actes du $32^{e}$ Congrès International de l'AFM. Lyon, France. Repéré à 
La gouvernance de Wikipédia :

élaboration de règles et théorie d'Ostrom

https://hal.archives-ouvertes.fr/hal-01341906/document

Kaplan, F. et Nova, N. (2016). Le miracle Wikipédia. Lausanne, Suisse : Presses polytechniques et universitaires romandes.

Konieczny, P. (2009). Governance, Organization, and Democracy on the Internet: The Iron Law and the Evolution of Wikipedia. Sociological Forum, 24(1), 162-192.

Konieczny, P. (2010). Adhocratic Governance in the Internet Age: A Case of Wikipedia. Journal of Information Technology \& Politics, 7(4), 263-283.

Langlais, P.-C. (2014). La négociation contre la démocratie : le cas Wikipédia. Négociations, 21(1), 21-34.

Langlais, P.-C. (2015). \{\{Référence nécessaire\}\} : l'émergence d'une norme wikipédienne (2003-2009). Dans L. Barbe, L. Merzeau et V. Schafer (dir.), Wikipédia objet scientifique non identifié (p. 77-90). Paris, France: Presses universitaires de Paris Ouest.

Le Crosnier, H. (2015). En communs: une introduction aux communs de la connaissance. Caen, France : C\&F éditions.

Lih, A. (2009). The Wikipedia Revolution: How a Bunch of Nobodies Created the World's Greatest Encyclopedia. Londres, Angleterre : Aurum Press Limited.

Ostrom, E. (2010). Gouvernance des biens communs : pour une nouvelle approche des ressources naturelle. Bruxelles, Belgique : De Boeck.

Pentzold, C. (2011). Imagining the Wikipedia community: What do Wikipedia authors mean when they write about their "community"?. New Media \& Society, 13(5), 704-721.

Russell, A. L. (2006). "Rough Consensus and Running Code" and the Internet-OSI Standards War. IEEE Annals of the History of Computing, 28(3), 48-61.

Sahut, G. (2014). "Citez vos sources »: archéologie d'une règle au cœur du savoir wikipédien (2002-2008). Études de communication, 42, 97-110. 
Sahut, G. (2015). Wikipédia, une encyclopédie collaborative en quête de crédibilité : le référencement en questions (Thèse de doctorat). Université de Toulouse, France.

Sahut, G. (2016). Construire une encyclopédie avec un wiki? Regards rétrospectifs sur la politique éditoriale de Wikipédia. I2D - Information, données \& documents, 53(4), 68-77.

Safner, R. (2016). Institutional entrepreneurship, Wikipedia, and the opportunity of the common. Journal of Institutional Economics, 1(4), 1-29.

Schroeder, A. et Wagner, C. (2012). Governance of open content creation: A conceptualization and analysis of control and guiding mechanisms in the open content domain. Journal of the American Society for Information Science and Technology, 63(10), 1947-1959.

Shaw, A. et Hill, B. M. (2014). Laboratories of oligarchy? How the iron law extends to peer production. Journal of Communication, 64(2), 215-238.

Viégas, F. B., Wattenberg, M., Kriss, J. et Van Ham, F. (2007). Talk before you type: Coordination in Wikipedia. Dans $40^{\text {th }}$ annual International Hawaii Conference on System Sciences (p. 78-87). Washington, DC : IEEE Computer Society Press.

Viégas, F. B., Wattenberg, M. et Mckeon, M. M. (2007). The hidden order of Wikipedia. Dans D. Schuler (dir.), Online communities and social computing (p. 445-454). Berlin, Allemagne : Springer-Verlag.

Zhou, T. (2011). Understanding online community user participation: A social influence perspective. Internet Research, 21(1), 67-81. 